\title{
Simplified Analytical Circuital Model of Damper Windings Exploiting Symmetries
}

\author{
S. Nuzzo, P. Bolognesi, M. Galea
}

\begin{abstract}
The damper windings of salient-pole, wound-field synchronous generators are currently attracting a renewed interest due to their impact on the machine efficiency and power quality, which was somewhat overlooked in the past. This paper focuses on the equivalent circuital modeling of damper windings. After recalling its main physical features, the general approach is first presented, permitting to define an equivalent circuit and to derive the related resistance and inductance matrixes whatever is the pattern of the cage bars. Further assumptions deriving from common or advanced features of damper windings are then analyzed, such as symmetrical, antisymmetrical, shifted and modulated position patterns. It is then shown how such assumptions can be exploited to simplify and shrink the equivalent circuital model of damper windings when analyzing realistic operating conditions.
\end{abstract}

Index Terms-Asymmetric Windings, Circuital Modelling, Damper Windings, Inductance Matrix, Permeability, Synchronous Generators, Winding Functions.

\section{INTRODUCTION}

$\mathrm{T}$ HE modelling aspects of damper windings (DWs) of wound-field synchronous generators (WFSGs) have been extensively discussed in literature [1]. In fact, thanks to the enduring history of use of such classical machines in a significant number of applications, both as motors and generators, DWs have been studied, modelled and optimized over the years. However, their preliminary "sizing" is still based mainly on empirical or semi-empirical equations [2-3] and the analysis of their effects on the machine performance is often accomplished through numerical simulation methods [4]. This is mainly due to the complexity of the phenomena associated with these windings, which makes difficult to effectively analyze their operation via analytical models, whereas a better accuracy is usually achieved using numerical simulation models.

Nevertheless, it is perceived that the circuital modelling of DWs has not been fully investigated. This paper thus focuses on this topic, aiming to provide useful considerations that can permit to analyze or design DWs in WFSGs. Firstly, the classical approach is recalled in its general form and then practical observations are presented with the aim of simplifying the model. An alternative way of modelling the DWs is then proposed and compared to the classical approach, highlighting differences and implications that

S. Nuzzo and M. Galea are with the Power Electronics, Machines and Control (PEMC) Group, University of Nottingham, Nottingham, UK (email: stefano.nuzzo@nottingham.ac.uk).

M. Galea is with the Power Electronics, Machines and Control (PEMC) Group, University of Nottingham Ningbo, Ningbo, China (e-mail: michael.galea@nottingham.edu.cn).

P. Bolognesi is with the Electrical Machines, Power Electronics and Drives Group, University of Pisa, Pisa, Italy (e-mail: p.bolognesi@ieee.org). inherently lead to further reduce the problem complexity. In this context, focus is given to the self- and mutualinductances involving the equivalent circuits of the DWs, evaluated by using the winding function (WF) theory [5]. The proposed approach is illustrated referring to a case study assumed as example.

\section{BACKGROUND}

Usually, synchronous machines are equipped with a DW. When implemented onto salient-pole WFSGs, it consists of longitudinal bars located in slots inside the pole shoes. The bars located inside each pole are connected together at each machine end. When such groups of bars are also sequentially connected at each side, a complete cage is obtained, otherwise an incomplete DW is achieved. Besides this structural-based differentiation, DWs can be classified also according to their resistance and inductance, whose values are chosen according to the application the synchronous machine is designed for. In most applications, the damper cage is designed for improving the machine transient behavior and thus strengthen the power system stability [6]. The effects of DWs on the sub-transient reactances and time constants, on the negative-sequence reactances and resistances and, in general terms, upon stability are wellknown and extensively studied [1, 3, 6]. Other reasons for equipping synchronous machines with DWs include a) to permit self-starting when directly supplied by the grid; b) to attenuate oscillations resulting from the pulsating torque provided by engines; c) to compensate for the negativesequence field component in 1-phase generators or in 3-phase generators supplying unbalanced loads; d) to reduce the stress on the insulation of the field winding during current surges in the armature windings, etc.

Besides these main purposes, actually DWs play a significant role also during "normal" steady-state conditions, both at no-load and on-load. In particular, currents may be induced in the damping bars both by the slot ripple (even at no-load) and the m.m.f. harmonics due to armature reaction. These phenomena are often particularly evident in mediumlarge size machines featuring open stator slots, where the losses associated to the currents induced in the DW can produce overheating issues [4]. On the other hand, these DW currents typically allow to attenuate such parasitic harmonics, thus indirectly improving the power quality in WFSGs. These aspects still represent a matter of on-going research, mainly due to the ever-increasing efficiency and power quality requirements. All the above is confirmed by the significant number of works recently published in this field [4, 7, 8].

Considering the challenges highlighted above, it is clear that a comprehensive and conceptual understanding of the 
electromagnetic phenomena related to the DWs can be very important when designing a WFSG. Therefore, an overview of the analytical modelling of such windings, based on the equivalent circuit methodology, is presented in this paper. Besides considering standard symmetrical DWs, the study investigates also non-standard configurations, including "shifted" and "modulated" damper cages.

\section{The Classical Circuital Modelling FOR SYMMETRICAL DWS}

As mentioned in Section II, from a structural point of view, DWs of salient-pole WFSGs can be classified according to their electrical connection at the end sides of the machine, thus resulting in complete or incomplete configurations. As the incomplete layout represents a particular case of the complete winding, the latter is first considered and its modelling presented in this section.

The end conductors in complete DWs are effectively low resistance paths, usually consisting of closed rings connecting the bars of all the poles. The classical, equivalent topology of $b$ bars connected by two end rings can be schematized as a planar net, ideally obtained by moving the front end ring on the same plane of the rear one while enlarging it, as qualitatively shown in Fig. 1 referring to an example featuring 4 poles and 6 bars per pole.

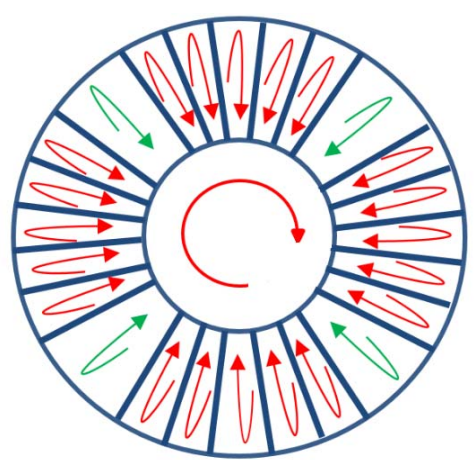

Fig. 1. Classical equivalent topology for a complete DW [8].

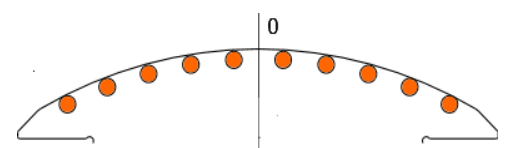

Fig. 2. 2-D cross section of one salient pole equipped with a DW with bars symmetrically displaced around the pole axis.

From an electromagnetic point of view, the set of currents flowing in the different branches of such net (bars and ring arcs) is equivalent to the set of the $b+1$ natural loop currents $i_{k}$ shown in Fig. 1, which may be assumed to have the same orientation (clockwise in the example). The currents singled out by the subscripts $k=1, \ldots, b$ flow sequentially in the $b$ loops delimited by two adjacent bars (numbered $k-1, k$ ) and by the inner and the outer arc sectors (labeled $I_{k}, O_{k}$ ) interposed between such bars. A further current named $i_{0}$ flows in the inmost loop representing the rear ring. In fact, the loop currents are independent from the point of view of Kirchoff first law, and determine in univocal way the currents flowing in each branch, as given in (1). In (1), $i_{b k}$ is the current flowing in the $k$-th bar, and $i_{l k}$ and $i_{O k}$ are the currents flowing in the inner and outer arc sectors, respectively.

$$
\begin{gathered}
i_{b k}(t)=i_{k}(t)-i_{k-1}(t) \\
i_{I k}(t)=i_{k}(t)-i_{0}(t) \\
i_{O k}(t)=i_{k}(t)
\end{gathered}
$$

On the other hand, the application of Kirchoff second law to the loops themselves permits to obtain a set of independent voltage equations which ensures that such law is verified also for any other closed path. Therefore, it is possible to model the DW as a set of $b+1$ phases whose terminals are shortcircuited, each one corresponding to one of the loops.

With respect to the resistive phenomena, considering as first case study a common symmetrical structure featuring an even distribution of identical damping bars along each of $2 p$ salient poles (as for example shown in Fig. 2), it can be noticed that a) all the $b$ bars have the same resistance value $\left.R_{b} ; \mathrm{b}\right)$ all the $2 b-4 p$ inner and outer arc sectors connecting bars within each pole group present the same resistance value $R_{s p}$; c) all the $4 p$ arc sectors connecting the bar groups of adjacent poles have the same resistance value $R_{d p}$. In order to simplify the analysis, as a first approximation $R_{s p}$ is considered equal to $R_{d p}$, i.e. assuming that $R_{s p}=R_{d p}=R_{a}$. Having defined these quantities, it becomes possible to write the Kirchhoff voltage law for each equivalent phase, as given in (2) for the generic $k^{\text {th }}$ natural loop delimited by bars, and in (3) for the end ring loop.

$$
\begin{aligned}
& v_{k}(t)=0=-R_{b} i_{k-1}(t)+2\left(R_{a}+R_{b}\right) i_{k}(t)-R_{b} i_{k+1}(t)-R_{a} i_{0}(t)+\frac{d \Phi_{k}(t)}{d t} \\
& v_{0}(t)=0=b R_{a} i_{0}(t)-R_{a} \sum_{k=1}^{b} i_{k}(t)+\frac{d \Psi_{0}(t)}{d t}
\end{aligned}
$$

In (2), $\psi_{k}$ is the flux linked to the generic loop/phase $k$, whereas in (3) $\psi_{0}$ represents the leakage flux linked to the rear ring of the DW. Flux and current directions are assumed to be coherent in each loop, according to the right-hand rule. It is worth grouping the voltage equations described above and relative to the $b+1$ equivalent DW loops in such a way to obtain the matrix form given in (4), where $\bar{i}(t)=\left[\begin{array}{lll}i_{1} \ldots i_{b} & i_{0}\end{array}\right]^{\mathrm{T}}$ contains all of the $b+1$ loop currents, and $\bar{\Psi}(t)=\left[\begin{array}{lll}\psi_{1} . . & \psi_{\mathrm{b}} & \psi_{0}\end{array}\right]^{\mathrm{T}}$ all the $b+1$ fluxes linked with the phases

$\overline{0}=\overline{R i}(t)+\frac{d \bar{\Psi}(t)}{d t}$

In the complete model of the machine, obviously (4) represents just part of the vector voltage equation, which also include 1 scalar equation per each other phase.

\section{A. The Resistance Matrix}

Considering first the resistive voltage term of (4), the square resistance matrix $R$, whose size is $(b+1) x(b+1)$ is easily derived from the expressions given in (2) and (3). Due to the dependency of the voltage drop in any bar or rear ring arc from both of the loop currents flowing in the two adjacent loops it belongs to, a non-diagonal form of $R$ is obtained as given in (5). The matrix remains symmetrical, as expected, but it features a tri-diagonal cyclic structure for the first $b$ rows-columns whereas the last row-column is fully non-zero. 


$$
R=\left[\begin{array}{ccccccc}
2\left(R_{a}+R_{b}\right) & -R_{b} & 0 & \ldots & 0 & -R_{b} & -R_{a} \\
-R_{b} & 2\left(R_{a}+R_{b}\right) & -R_{b} & 0 & \ldots & 0 & -R a \\
0 & -R_{b} & \ldots & \ldots & \ldots & \ldots & \ldots \\
\ldots & 0 & \ldots & \ldots & \ldots & 0 & \ldots \\
0 & \ldots & \ldots & \ldots & \ldots & -R_{b} & -R_{a} \\
-R_{b} & 0 & \ldots & 0 & -R_{b} & 2\left(R_{a}+R_{b}\right) & -R a \\
-R_{a} & -R_{a} & \ldots & \ldots & -R_{a} & -R_{a} & b R_{a}
\end{array}\right]
$$

\section{B. The Inductance Matrix}

As a first approach aimed to minimize the complexity of the circuital model, in this paper the ferromagnetic materials are considered linear. Under such hypothesis, the electromagnetic voltage term in (4) can be elaborated as in (6), where the square inductance matrix $L$ referred to the $b+1$ equivalent DW phases is introduced, assuming that it depends only on the angular position $\alpha$ of rotor vs. stator. In (6), also the flux $\overline{\Psi_{a f}}(t)$ linked with the DWs due to the field and armature windings is introduced.

$$
\frac{d \bar{\Psi}(t)}{d t}=\left(\left.\frac{\partial L(\alpha)}{\partial \alpha}\right|_{\alpha(t)} \bar{i}(t)\right) \frac{d \alpha(t)}{d t}+L(\alpha(t)) \frac{d \bar{i}(t)}{d t}+\frac{d \overline{\Psi_{a f}}(t)}{d t}
$$

Besides the assumption of linear characteristics for the magnetic materials, it is convenient to assume that the mutual influence between the main and the secondary field lines can be neglected, permitting to separately analyze such phenomena. Also, the analysis can be carried out by considering only to the main flux tubes, as these are responsible for the electromechanical energy transformation, thus leading to ignore the leakage flux contributions. This is a fairly realistic assumption, unless niche applications are considered [9]. On the other hand, the flux $\psi_{0}$ linked to the rear end ring can be obviously considered as relatively weak leakage flux, as the related field lines develop in the axial direction. Hence, the related electromagnetic voltage in (3) is negligible, thus leading to an additional interesting conclusion, expressed by (7).

$$
i_{0}(t)=\frac{1}{b} \sum_{k=1}^{b} i_{k}(t)
$$

Considering the above assumptions and implications, it is worth to express $L(\alpha)$ as only referred to the main flux tubes, in order to exploit and carry out considerations on all its elements. The general expression given in (8) and obtained in [10] can be used for such purpose.

$$
\begin{gathered}
L(\alpha)=l \cdot \int_{0}^{1} \mu_{E}(\lambda, \alpha) \cdot \overline{N_{E}}(\lambda, \alpha) \cdot{\overline{N_{E}}}^{T}(\lambda, \alpha) \cdot d \lambda \\
\overline{N_{E}}(\lambda, \alpha)=\bar{N}(\lambda, \alpha)-\int_{0}^{1} \frac{\mu_{E}(\lambda, \alpha)}{1} \mu_{E}(\lambda, \alpha) \cdot d \lambda \\
0
\end{gathered}
$$

In (8), $l$ is the axial length of the machine, $\lambda$ is the normalized tangential coordinate mapping the whole airgap, $\mu_{E}(\lambda, \alpha)$ is the equivalent permeability function summarizing the effects of airgap size and shape and $\overline{N_{E}}(\lambda, \alpha)$ is the vector containing the equivalent winding functions (EWFs). The latter is defined in (9), as the difference between the vector $\bar{N}(\lambda, \alpha)$ containing the WFs of the machine phases and its weighted average value calculated vs. $\mu_{E}(\lambda, \alpha)$.

In general, in a salient-pole WFSG all the WFs are of the type shown in Fig. 3, i.e. simple pulse-like stepwise waveforms. The EWFs keep then the same shape, as also shown in red in Fig. 3. The interval separating the fronts in the function is determined by the distance between the bars delimiting the loop under examination. Therefore, it is usually smaller for the $b-2 p$ loops delimited by bars embedded in the same pole and larger for the $2 p$ loops, shown in green in Fig. 1, which are delimited by the facing end bars belonging to two adjacent poles

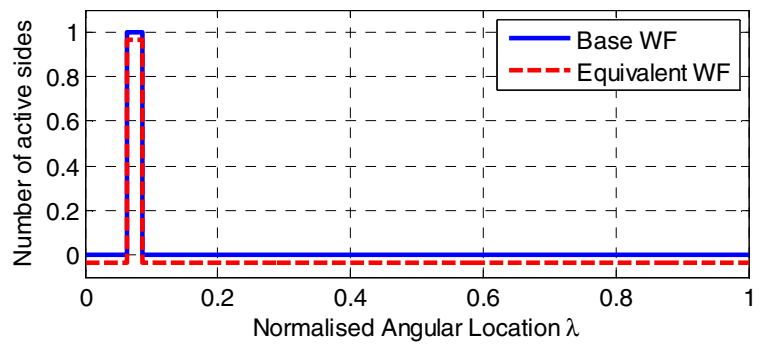

Fig. 3. WF and EWF for a generic damper cage loop.

Considering the above, the DW inductances calculated as in (8) lead to the following observations:

1) the self-inductance $L_{k}(\alpha)$ of any loop phase assumes a positive value whatever is the trend of $\mu_{E}(\lambda, \alpha)$; as expected; such value is almost constant vs. $\alpha$, as any variation is only due to the stator slot openings.

2) The mutual-inductance $L_{i j}(\alpha)$ between the $i^{\text {th }}$ and the $j^{\text {th }}$ loop phases assumes a negative value for any $\alpha$ whatever is the trend of $\mu_{E}(\lambda, \alpha)$. As well as for the self-inductances, a slight dependence of the mutual inductances on the position $\alpha$ can be detected due to the slot openings.

Considering all the above, it can be concluded that in general all the elements of $L$ are dependent on $\alpha$ and different from each other; anyway, the matrix has to be symmetrical to comply with the energy conservation principle.

\section{MODEL SimplifiCATIONS}

In this section, some simplifications resulting from practical considerations are applied to the circuital model presented in its general framework in Section III.

The first simplification consists in considering a null value of the loop current $i_{0}$ flowing in the inner loop of Fig. 1. In fact, the symmetry of the machine and cage structure that is commonly adopted makes such hypothesis fairly realistic, as for each bar loop another one located just 1 pole pitch apart is often found, meaning that the related loop currents can be expected to be almost opposite in normal operating conditions. Therefore, such loop current and the corresponding voltage equation in (4) can be neglected, resulting in a reduction of the matrixes $R$ and $L$ to the $b x b$ subparts obtained from (5) by deleting the row and column 
relative to $i_{0}$. This results in a tridiagonal form of the resistance matrix and in a full form of the inductance matrix. Also, this hypothesis means that the first member of (7) is equal to zero, leading to obtain a null value of the total sum of the currents flowing inside the bars.

Besides the aspects mentioned above, additional simplifications can be applied to the circuital model of the DW. In particular, the end connections between any couple of adjacent bars inside any salient pole typically present an equivalent resistance $R_{a}$ that is much lower than the resistance $R_{b}$ of the bars. Therefore, the ring sector resistance $R_{a}$ can be considered as negligible in (5), meaning that all the elements in the main diagonal can be assumed just equal to $2 R_{b}$. On the other hand, the end connections linking any couple of bars embedded in different poles either consist of higher resistance paths (e.g. when v-shaped end plates are used) or may be even not installed at all, making the group bars in each salient pole almost insulated. In this case, the DW is labelled as incomplete, resulting in the missing of $2 p$ equivalent loop phases as shown in Fig. 4.

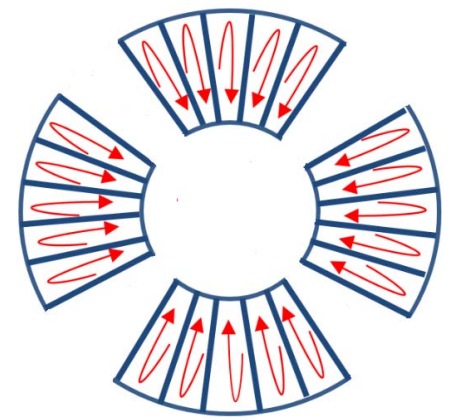

Fig. 4. Classical equivalent topology for an incomplete DW [8].

When the cage is incomplete, the number of loops to be considered is then reduced, meaning that the dimensions of $R$ and $L$ matrixes become $(b-2 p) x(b-2 p)$. Also, as the loop currents flowing in any pole are not correlated to the currents circulating in the equivalent phases of the other poles, the matrix $R$ turns out to be composed of $2 p$ submatrices, whose elements in the positions " $1, b / 2 p-1$ " and " $b / 2 p-1,1$ " become null in this new configuration.

Furthermore, usually in small-mid size WFSGs the following additional hypotheses can be assumed at least as a first approximation: a) negligible effects of stator slot openings for the definition of the equivalent permeability function; b) constant and equal airgap thickness along the tangential development of each salient pole. Under such hypotheses, the following implications on the inductance matrix related to the equivalent DW phases can be observed:

1) All the self- and mutual-inductances are constant quantities with respect to $\alpha$.

2) All of the $b-2 p$ self-inductances of the loops including bars located within the same pole are equal to a common value $L_{b l}$;

3) In complete DWs, all of $2 p$ self-inductances of the loops including bars located in adjacent poles are equal to a common value $L_{b 2}$, different from $L_{b 1}$.

4) The $(b-2 p) x(b-2 p-1)$ mutual-inductances between the equivalent phases delimited by bars located inside the same pole are equal to a common value $L_{m l}$.

$5)$ In complete DWs, also the $(2 p) x(2 p-1)$ mutualinductances between the loop phases located across adjacent poles are equal to a common value $L_{m 2}$.

6) In complete DWs, also the $(2 p) x(b-2 p)$ mutualinductances between equivalent phases of different span are equal to a common value $L_{m 3}$.

In order to summarize the assumptions and implications described above, it is worth to show the updated versions of the matrices $R$ and $L$, considering for example an incomplete DW. In (10), the resistance submatrix relative to one pole is shown, whereas the inductance matrix is given in (11).

$$
\begin{aligned}
R & =\left[\begin{array}{ccccc}
2 R_{b} & -R_{b} & 0 & \ldots & 0 \\
-R_{b} & 2 R_{b} & -R_{b} & 0 & \ldots \\
0 & -R_{b} & \ldots & \ldots & 0 \\
\ldots & 0 & \ldots & \ldots & -R_{b} \\
0 & \ldots & 0 & -R_{b} & 2 R_{b}
\end{array}\right] \\
L & =\left[\begin{array}{ccccc}
L_{b 1} & L_{m 1} & \ldots & \ldots & L_{m 1} \\
L_{m 1} & L_{b 1} & L_{m 1} & \ldots & \ldots \\
\ldots & L_{m 1} & \ldots & \ldots & \ldots \\
\ldots & \ldots & \ldots & \ldots & L_{m 1} \\
L_{m 1} & \ldots & \ldots & L_{m 1} & L_{b 1}
\end{array}\right]
\end{aligned}
$$

\section{Alternative Circuital Model Applied to VARious DW TOPOLOGIES}

In the previous sections, first the circuital modelling of DWs of salient-pole WFSGs has been presented in its most general framework (see Section III). Then, the simplifications that can be often assumed considering typical characteristics of the machine have been discussed (see Section IV), leading to the reduced resistance and inductance matrices shown in (10) and (11). This section describes an alternative way of modelling such a rotor winding, resulting from the exploitation of all the symmetries often observed in a WFSG. Such approach is first applied to symmetrical DWs, showing how it is possible to significantly decrease the complexity of the system (4) with respect to the classical approach. The alternative model is then properly adapted for modelling shifted and modulated DWs, in order to highlight the general implications on the complexity of the whole system and on the resistance and inductance matrices.

\section{A. Even-Symmetrical Windings}

In their most popular configuration, small-mid size WFGSs feature a full cyclic structural symmetry according to the number of pole pairs, meaning that at least each pair of rotor poles are identical also with respect to the deployment of the DW. In particular, the poles feature a symmetrical distribution of bars with respect to the pole axis as shown in Fig. 2. Therefore, although the DW structure could theoretically permit a more general behavior, in practice only operating conditions strictly related to the cyclic symmetry above recalled are likely to be observed and thus interesting to be analyzed. This means that also the patterns of the currents flowing inside the bars of the $p$ angular sectors can be assumed to be identical, just as for the other windings. It 
becomes then possible to further simplify the model by embedding such constraint in the equivalent circuit, assuming that all the $p$ loops located in the corresponding positions of homologous poles are connected in a concurrent closed series, as this is just equivalent to have each of them individually shorted. In this way, the number of equivalent phases representing the DW via such compound loops is reduced by a factor just equal to $p$. This concept is illustrated in Fig. 5 for one equivalent phase of the 4-pole case study. The WF of this series-connected phase can be represented by a sequence of $p$ positive pulse-like waveforms, as shown in Fig. 6. In the same figure, the EWF of this updated loop phase calculated as in (9) is also shown in red.

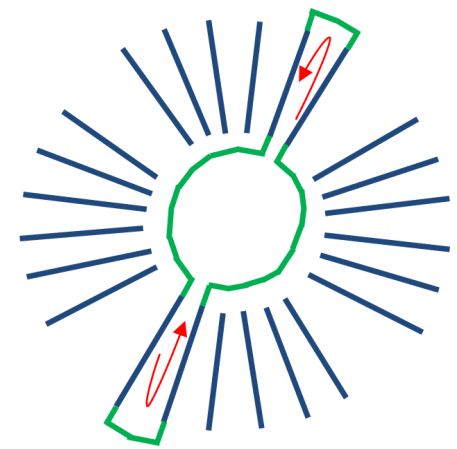

Fig. 5. Compound loop in the DW of a 4-poles cyclic machine.

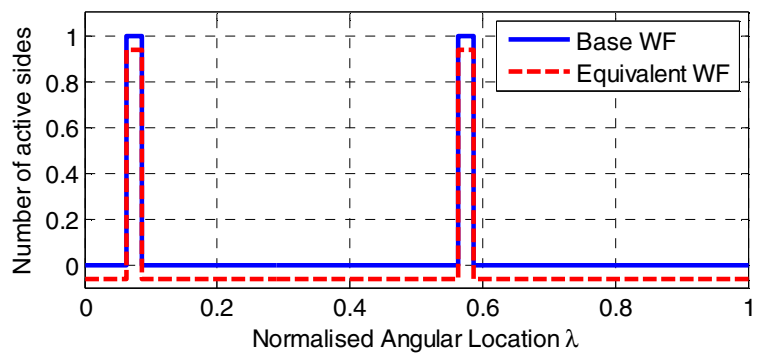

Fig. 6. Base and equivalent winding functions for the series-connected equivalent phase loop shown in Fig. 5.

Taking into account the practical considerations discussed in Section IV, the Kirchhoff voltage law can be written for each new equivalent phase as in (12).

$$
v_{k}(t)=0=-p R_{b} i_{k-1}(t)+2 p R_{b} i_{k}(t)-p R_{b} i_{k+1}(t)+\frac{d \Psi_{k}(t)}{d t}
$$

It can be easily deduced that the new versions of $R$ and $L$ are of the same type of those shown in (10) and (11) respectively, but with smaller dimensions as the number of equivalent phases is reduced to $b / p$ for complete DWs and $b / p-2$ for incomplete DWs (obviously neglecting the rear end ring loop). In particular, all the elements of $R$ and $L$ in the updated DW model present a value $p$ times higher than that of the classical model.

\section{B. Odd-Symmetrical Windings}

In addition to the cyclic symmetry previously recalled, an odd cyclic symmetry is often present in the electromagnetic structure of the machine. In fact, usually the stator and field windings feature alternating WFs (apart from an irrelevant offset), whereas the damping bars are located in identical positions inside each pole. In such case, although again the DW structure could theoretically permit a general behaviour, the analysis can be realistically limited to the operating conditions that comply with such symmetry. It can be thus assumed that the current patterns related to the bars located in the 2 sequences of homologous poles are exactly opposite. Such assumption permits to further reduce the size of the model by embedding the above constraint in the equivalent the above constraint. This is achieved by connecting in series all the DW loops located in corresponding positions inside the poles, obviously with appropriate polarity to ensure that the new arrangement ensures the zeroing of the equivalent phase voltages. An example of such connection is shown in Fig. 7: the number of equivalent phases representing these compound loops is then further halved down to $b / 2 p-1$ for incomplete DWs and $b / 2 p$ for complete DWs.

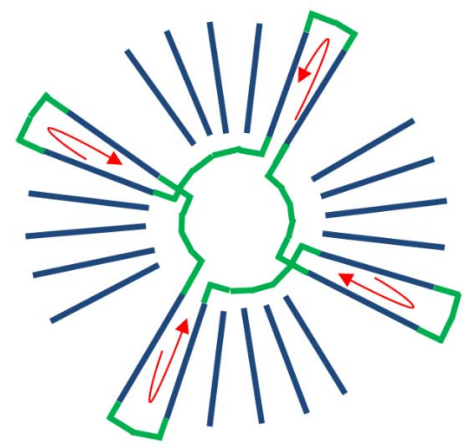

Fig. 7. Compound loop in the DW for symmetrical DWs [8].

The Kirchhoff voltage law for any of the equivalent loop phases can be written as in (12) for the series-connected loop, but with the second member of such equation just multiplied by 2 , as the number of bars-per-phase involved in this model is just 2 times higher than that of the previous case. For incomplete DWs the resistance matrix results then as shown in (13). In case that a complete cage is considered, the elements in the corner positions " $1, b / 2 p$ " and " $b / 2 p, 1$ " are equal to $+2 p R_{b}$ as the currents $i_{1}$ and $i_{b / 2 p}$ flow in the same direction inside the bars shared by the relevant loop phases.

$$
R=\left[\begin{array}{ccccc}
4 p R_{b} & -2 p R_{b} & 0 & \ldots & 0 \\
-2 p R_{b} & 4 p R_{b} & -2 p R_{b} & 0 & \ldots \\
0 & -2 p R_{b} & \ldots & \ldots & 0 \\
\ldots & 0 & \ldots & \ldots & -2 p R_{b} \\
0 & \ldots & 0 & -2 p R_{b} & 4 p R_{b}
\end{array}\right]
$$

Referring to the electromagnetic aspects, it can be noticed that the WFs of each of the new equivalent phases representing the $2 p$ compound loops is composed of an even sequence of $2 p$ positive-negative pulse-like waveforms. Such waveform features inherently a periodical alternating trend with period equal to 2 pole pitches. Considering that the equivalent permeability function of any normal symmetric machine is either constant or features a period just equal to 1 pole pitch, it can be concluded that the weighted average of the base WF is null in any case, meaning that the EWF, calculated as in (9), will result just equal to the base WF itself. This implies that the EWFs related to different compound phases will not share any interval where both are non-zero. Therefore, according to (8), their mutual inductances result equal to zero. This concept is depicted in Fig. 8, where the EWFs $N_{E j}$ and $N_{E k}$ of 2 generic equivalent 
phases can be observed. Therefore, it can concluded that, by using the series/antiseries connected phases, the problem complexity is further significantly reduced, as the inductance matrix $L$ becomes a diagonal matrix as shown in (14).

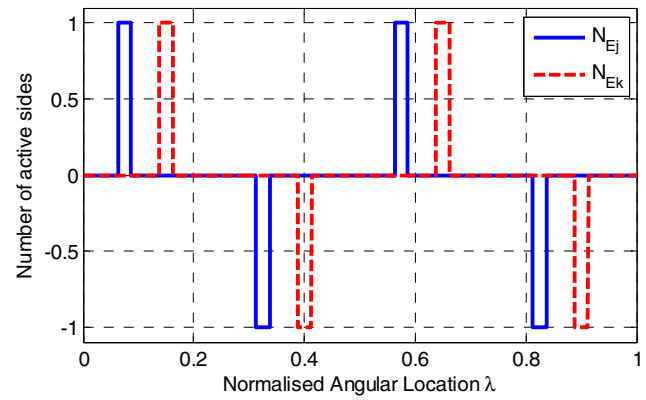

Fig. 8. Equivalent winding functions of two generic series/antiseriesconnected cage loop phases for odd-symmetrical DWs

$$
L=\left[\begin{array}{cccc}
L_{b} & 0 & \ldots & 0 \\
0 & \ldots & \ldots & \ldots \\
\ldots & \ldots & \ldots & 0 \\
0 & \ldots & 0 & L_{b}
\end{array}\right]
$$

In (14), the assumptions discussed in Section IV relative to the absence of slot opening effects and to the constancy of the airgap are taken into account: therefore, the elements on the main diagonal present the same common value $L_{b}$ and are constant quantities with respect to the rotor position $\alpha$.

The series/antiseries compound representation of the DW loops allows then to obtain significant simplifications with respect to the general model described in Section III, mainly due to a) a significant reduction of the system dimensions proportional to the number of pole pairs; b) the total elimination of the magnetic couplings between the equivalent DW phases. However, the above conclusions are applicable only to odd-symmetrical DWs. Although this represents the most popular configuration, there exist some alternative patterns that cannot be entirely modelled as described in this section. The modelling of such alternative patterns will be the focus of the next sections.

\section{Shifted Damper Windings}

The shifted damper cage [12] represents a departure from the standard symmetrical configuration. It consists in symmetrically displacing the groups of bars located in the odd and even salient pole sequences around the polar axis in alternating directions, as shown in Fig. 9. The shifted DW is usually implemented to reduce the output voltage distortion, however coming at the cost of larger induced currents and associated losses in the bars [7].

From a modelling point of view, when the armature and field windings comply with the even structural symmetry considered in Section V.A, then the shifted DW pattern also complies with such hypothesis. On the other hand, it does not fulfil the odd structural symmetry described in Section V.B. Therefore, the series compound configuration derived in Section V.A and illustrated in Fig. 5 can be adopted, meaning that compound equivalent phases can be considered and all of the conclusions previously drawn can be applied. In particular, base and equivalent WFs are of the same type of those shown in Fig. 6.

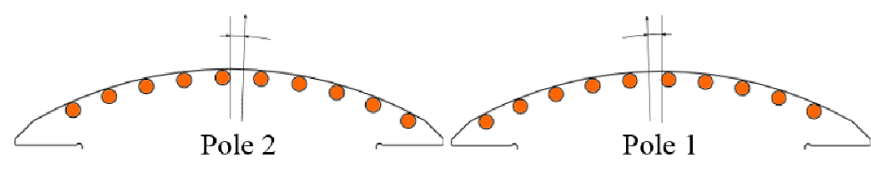

Fig. 9. Example of salient pole pair equipped with a shifted DW.

\section{Modulated Damper Windings}

The modulated DW is a concept recently proposed to overcome both the challenges related to the WFSGs' voltage harmonic content and to the high bar losses [7]. The pattern proposed in [7] is shown in Fig. 10, where the unconventional asymmetrical distribution of the bars can be observed for 1 salient pole.

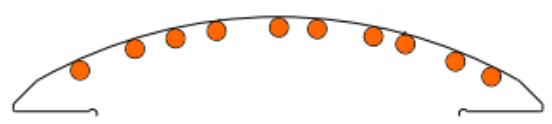

Fig. 10. Salient pole equipped with a modulated DW [7].

Such DW structure complies with the odd symmetry conditions assumed in Section V.B, meaning that the series/antiseries compound phase representation derived and illustrated in Fig. 7 and Fig. 8 can be applied. Nevertheless, the EWFs of the various compound loop phases related to adjacent bars within the same poles do not feature the same distance between the fronts due to the irregular bar pitch. An example of 2 WFs of a modulated damper cage is illustrated in Fig. 11. As a consequence of this concept, while the resistive phenomena are not modified with respect to the symmetrical scenario (obviously under the assumption of negligible bar-to-bar end resistances), the self-inductances $L_{b i}$ (with $i=1, \ldots, n$ ) of the various loop phases may be different to each other. Therefore, the structure of matrix $R$ is just the same as in (13), while the matrix $L$ results as in (15). Therefore, the number of equivalent phases is equal to $n=b / 2 p-1$ for incomplete DWs and $n=b / 2 p$ for complete DWs when neglecting the loop relative to the rear end ring.

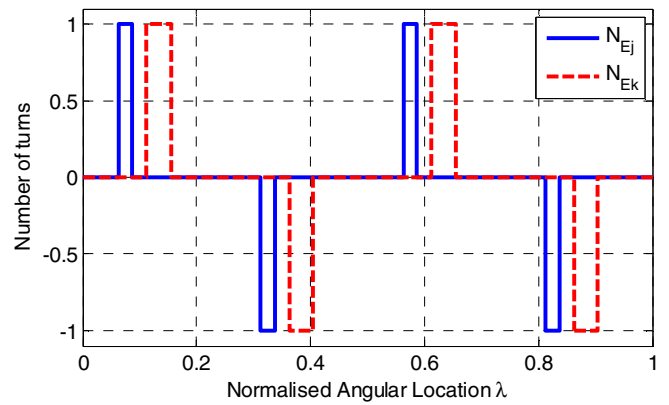

Fig. 11. Equivalent winding functions of two generic series/antiseries connected cage loop phases for modulated DWs.

$$
L=\left[\begin{array}{cccc}
L_{b 1} & 0 & \ldots & 0 \\
0 & L_{b 2} & \ldots & \ldots \\
\ldots & \ldots & \ldots & 0 \\
0 & \ldots & 0 & L_{b n}
\end{array}\right]
$$

It is worth mentioning that the modulated DW concept can be implemented also in a more general way than proposed in [7]. In fact, a constraint-less optimization of the positions of the cage bars could lead to a completely irregular distribution over the whole round turn. In such scenario, obviously only the general model described in Section III could be adopted. 


\section{SUMMARIZING REMARKS - A CASE STUDY}

As a vehicle to summarize all the modelling aspects and the relevant implications discussed in this paper, a typical 3phase salient-pole WFSG is considered. The machine features 4 poles and 6 damping bars per pole, symmetrically displaced around the polar axes (as that shown in Fig. 2). The classical circuital model of this case study as discussed in Section III comprises $1+3+(6 * 4+1)=29$ equivalent phases.

Another important design aspect to mention for the scope of this section is that the DW end connections are realized through press-plate laminations and thus the damper cage can be classified as incomplete. Considering the inherent implications discussed in Section IV, in this case the number of equivalent phases becomes $1+3+(6 * 4-4)=24$.

The number of phases and the complexity of the circuital model are further reduced due to the odd-symmetrical layout featured by the DW of the considered WFSG. Therefore, the machine now comprises $1+3+(24 / 4-1)=9$ equivalent phases and the mutual inductances between the cage loops become all null, as discussed in Section V.B.

When the DW was to be shifted as in Fig. 9, then an evensymmetrical layout would be achieved and the circuital model would comply with the observations presented in Section V.A, thus resulting in $1+3+(24 / 2-2)=14$ equivalent phases. Finally, if a modulated DW was to be implemented in such WFSG, this could feature either: a) an odd-symmetrical layout, leading to 9 equivalent phases with null mutual inductances and different self-inductances relative to the damper cage loops; or b) an even-symmetrical layout, resulting in 14 equivalent phases; or c) a non-symmetrical configuration, which could be analyzed through the general model recalled in Section III.

\section{CONCLUSION}

This paper presented a journey through the circuital modelling aspects of damper windings of salient-pole synchronous generators.

First, the general approach has been described and applied to the most popular damper cage configuration consisting of bars symmetrically displaced around the salient-poles axes.

Practical considerations have been then taken into account aiming to reduce the overall system complexity and highlighting the major differences between complete and incomplete damper windings.

A more effective way of modelling such winding has been presented in the second part of the paper. The proposed approach allows to significantly reduce the model dimension by properly exploiting the symmetries relevant to the damper winding. The model complexity is further reduced thanks to the zeroing of the mutual inductances between cage loops.

Non-standard damper winding patterns including shifted and modulated bars configurations have been also analyzed, highlighting the differences in the modeling with respect to the more common configurations.

Finally, an application example of the circuital model simplifications discussed in this paper has been presented referring to an existing industrial WFSG.

\section{REFERENCES}

[1] C. Concordia, Synchronous Machines - Theory and Performance: Chapman and Hall, Ltd., 1952.

[2] J. Pyrhonen, T. Jokinen, and V. Hrabovcova, Design of rotating electrical machines. Somerset, GB: Wiley, 2013.

[3] I. Boldea, The Electric Generators Handbook - Synchronous Generators. Boca Raton, FL: CRC Press, Taylor \& Francis Group, 2006.

[4] S. Nuzzo, M. Galea, C. Gerada, D. Gerada, A. Mebarki, and N. L. Brown, "Damper cage loss reduction and no-load voltage THD improvements in salient-pole synchronous generators," in 8th IET Int. Conf. Power Electron. Mach. Drives (PEMD), Apr. 2016, pp. 1-7.

[5] T. A. Lipo, Analysis of synchronous machines: CRC Press Taylor \& Francis Group, 2012.

[6] E. Kimbark, Power System Stability-Volume 1: Wiley-IEEE Press, Chapter XIV, 1995.

[7] S. Nuzzo, M. Degano, M. Galea, C. Gerada, N. Brown, and D. Gerada, "Improved Damper Cage Design for Salient-Pole Synchronous Generators," IEEE Trans. Ind. Electron., vol. 64, pp. 1958-1970, 2017.

[8] S. Nuzzo, P. Bolognesi, M. Galea, and C. Gerada, "An hybrid analytical-numerical approach for the analysis of salient-pole synchronous generators with a symmetrical damper cage," in IEEE International Electric Machines and Drives Conference (IEMDC), Miami, FL, USA, 2017.

[9] P. Giangrande, F. Cupertino and G. Pellegrino, "Modelling of linear motor end-effects for saliency based sensorless control", in $2^{\text {nd }}$ IEEE Energy Conversion Congress and Exposition, Atlanta, Georgia, USA

[10] P. Bolognesi, "A mid-complexity analysis of long-drum-type electric machines suitable for circuital modelling," Proc. of ICEM 2008 Conf., Vilamoura, Sep. 2008.

[11] A. Biebighauser and A. Ghita, "Rotor for a rotating electrical machine," UK Patent WO 2014/202985 A2, Dec. 24, 2014.

\section{BIOGRAPHIES}

Stefano Nuzzo received the B.Sc. and M.Sc. degrees in Electrical Engineering from the University of Pisa, Pisa, Italy, in 2011 and 2014, respectively. He received his Ph.D. degree in Electrical Machine Design in 2018 from the University of Nottingham, Nottingham, U.K., where is currently working as a Research Fellow within the Power Electronics, Machines and Control (PEMC) Group. His main research interests are the modelling, analysis and optimization of electrical machines and drives, with focus on salient-pole synchronous generators and brushless excitation systems for industrial power generation application.

Paolo Bolognesi received his M.Sc. and Ph.D. degrees in Electrical Engineering from the University of Pisa in 1995 and 1999, respectively. He joined then the University of Pisa as a post-doc junior researcher and finally as a Senior Researcher and Appointed Professor since 2001. His research interests span from modeling, analysis and design of electromagnetic devices, including unconventional electric machines and electromechanical actuators, to innovative topologies and modulation methods for static converters, also for applications related to electric and hybrid vehicles.

Michael Galea received his $\mathrm{PhD}$ in electrical machines from the University of Nottingham, UK, where he also worked as a Research Fellow. He is currently the Head of the School of Aerospace in the University of Nottingham, Ningbo, China, where he is also the Director of Aerospace. He currently lectures in Electrical Drives and in Aerospace Systems Integration and manages a number of diverse projects related to the more electric aircraft and electrified propulsion. His main research interests are design, analysis and thermal management of electrical machines and drives, the more electric aircraft and electrified and hybrid propulsion. 